\title{
Investigation of penetrative convection in stratified fluids through 3D-PTV
}

\author{
Valentina Dore · Monica Moroni · Martin Le Menach • \\ Antonio Cenedese
}

Received: 2 October 2008/Revised: 6 July 2009/Accepted: 8 July 2009/Published online: 31 July 2009

(c) The Author(s) 2009. This article is published with open access at Springerlink.com

\begin{abstract}
Testing existing theories of flow and transport in stably stratified fluids when "penetrative convection" occurs requires experimental methods capable of fully three-dimensional descriptions of both the Eulerian velocity field and Lagrangian particle trajectories. Boundary layer experiments utilizing a photogrammetric three-camera three-dimensional Particle Tracking Velocimetry (3D-PTV) technique were performed on a laboratory model which simulated the evolution of the mixing layer in a stably stratified fluid body by heating a tank filled with water from below. A combination of image- and object space-based information was employed to establish the spatio-temporal correspondences between particle positions at consecutive time steps. An appropriate calibration procedure for the stereoscopic system provides the intrinsic and extrinsic parameters of the stereoscopic system to be used for determining the correspondence of points in the object or world reference frame and in the image reference frame. The photogrammetric principles used by 3D-PTV are described. First, the fundamental mathematical model of the collinearity condition and its extensions are explained. Then, the epipolar line intersection method built upon multicamera correspondences (structure-from-stereo) is
\end{abstract}

V. Dore $(\bowtie) \cdot$ M. Moroni · A. Cenedese

DITS, Sapienza University of Rome, Rome, Italy

e-mail: valentina.dore@uniroma1.it

M. Moroni

e-mail: monica.moroni@uniroma1.it

A. Cenedese

e-mail: antonio.cenedese@uniroma1.it

M. Le Menach

ENSMA, Poitiers, France

e-mail: lemenacm@etu.ensma.fr discussed as well as the procedure of reconstruction of particle trajectories in a single step by conjunctly establishing the spatial and temporal correspondences between particle images. Tests on simulated data were performed as well as experimental data in order to ensure the method's operability and robustness. The reconstructed velocity fields are compared to other laboratory investigations and field data.

\section{Introduction}

A penetrative convective flow occurs in the atmosphere when solar heating creates an unstable boundary layer at the earth's surface. In lakes and oceans, an analogous phenomenon occurs during strong surface cooling, starting from the upper free surface and penetrating downward with time, also known as turn-over. When penetrative convection occurs, the flow field is organized into domes or spatial regions with significant vertical motion (Stull 1988; Moroni and Cenedese 2006). In the atmosphere, the domes spring up from the earth surface and stress the stable layer above creating internal waves over the mixing layer. In lakes and oceans, domes start at the cooler surface and stress the stable layer below. Convection is initially organized in persistent coherent structures, but later the flow becomes turbulent and the structures form and break randomly in space. Within the unstable layer, the fluid temperature, density and velocity change rapidly with time. The dynamics of penetrative convection in nature influence the transport and mixing of stratified fluids with implications for air and water quality, climate change and adsorption of UV radiation (Haynes and Shuckburg 2000).

When $2 \mathrm{D}$ techniques are employed to detect the velocity field, the flow is illuminated with a thin light sheet and only 
the velocity components within this sheet can be evaluated. Although some methods do exist for reconstructing 3D velocities in a point (3D laser Doppler anemometry; Hinsch and Hinrichs 1996) or plane (3D stereo-PIV (Particle Image Velocimetry); Stuer et al. 1999), only a fully 3D technique based on the illumination of a flow volume rather than a flow sheet will give the information needed to construct the instantaneous 3D velocity fields. A number of imagingbased measurement techniques exist for determining 3D velocity fields in an observation volume. Among these are:

- "scanning" techniques (Guezennec et al. 1994; Moroni and Cushman 2001);

- holographic techniques (Hinsch and Hinrichs 1996; Zhang et al. 1997);

- defocusing techniques (Willert and Gharib 1992);

- photogrammetric techniques (three-dimensional Particle Tracking Velocimetry, 3D-PTV; Maas 1992; Kasagi and Nishino 1990).

We will focus on 3D-PTV which is a 3D extension of the 2D particle-tracking method. Instead of tracking particles in a thin light sheet, the particles are now tracked in an illuminated volume. 3D-PTV is based on reconstructing 3D trajectories of reflecting tracer particles through a photogrammetric recording of image sequences. The 3D particle trajectories obtained can be used to calculate the $3 \mathrm{D}$ velocity field. Because the actual path of the particles is analyzed, particle-tracking techniques are generally more accurate than PIV-based techniques (Cowen and Monismith 1997; Moroni and Cenedese 2005).

The technique of 3D particle tracking was introduced by Chang and Taterson (1983) and further developed by (among others) Maas et al. (1993). Suzuki et al. (2000) applied 3D-PTV to simultaneous measurements of fluid and dispersed phases in a turbulent water channel flow and clarify the mutual interaction between near-wall coherent structures and solid particles. Instantaneous 3D vectors of tracer and solid particles were obtained from four successive sets of particle images. Kieft et al. (2002) applied a 3D-PTV algorithm to the wake flow behind a heated cylinder. The performance of the 3D particle localization and tracking technique was studied and tested with respect to its accuracy. Both for the calibration and the accuracy tests, synthetic data were created by traversing a $2 \mathrm{D}$ grid through the measuring volume. The method assumes that a particle in a camera image will have a position in the world coordinate system somewhere along the particle projection line. The points in space where the lines from the different cameras cross are the possible positions of the particles. Due to optical disturbances, random camera noise and errors in the calibration data, an exact crossing of the lines is unlikely and a line crossing is detected when the minimum distance between the lines is smaller than a certain critical value. Doh et al. (2004) describe 3D-PTV experiments investigating the structure of a sphere wake. To obtain the three-dimensional velocity vectors, camera parameters were obtained in advance. Next, the position of each particle was detected and finally the three-dimensional velocity vectors were calculated.

Here, we present the application of 3D-PTV to the study of penetrative convection in a stratified fluid heated from below. The 3D-PTV system has been designed with the following capabilities: image a volume far away the boundary walls, lengthen the trajectories, and improve the accuracy of the procedure through a careful test on synthetically generated data. A physically based photogrammetric calibration of the stereoscopic arrangement was employed and its accuracy tested. The effects of multimedia geometry on calibration parameters are taken into account. The combination of image- and object space-based information was employed to establish the correspondences between particle positions (structurefrom-stereo reconstruction). A particle-tracking algorithm was then employed to reconstruct 3D trajectories.

The paper is organized as follows. In Sect. 2, the experimental set-up and procedures are described. Section 3 presents a thorough discussion of the photogrammetric particle-tracking algorithm showing the details of the calibration procedure and the structure-from-stereo analysis. The performance of the matching algorithm applied to synthetic data as well as the results of the fluid flow measurements are discussed in Sect. 4. Finally, we present some conclusions.

\section{Experimental set-up}

The laboratory model consists of a convection chamber containing an initially stable, density stratified fluid, which was then heated from below to cause destabilization and penetrative convection.

The test section is a tank with a square base $\left(0.41 \times 0.41 \mathrm{~m}^{2}\right)$ and $0.40 \mathrm{~m}$ high (Fig. 1). Its lateral sides are insulated by $0.03 \mathrm{~m}$ thick removable polystyrene sheets. When images are acquired, the insulation on the sides facing the cameras is removed. Distilled water is used for the fluid phase and pollen particles of about $40 \mu \mathrm{m}$ mean diameter and $1.06 \mathrm{~g} / \mathrm{cm}^{3}$ density (Moroni and Cenedese 2005) are used for the passive tracer to reconstruct particle trajectories. A diffuser, which also acts to insulate the upper surface since it is attached to a polystyrene sheet, floats on the surface of the water as it fills the tank. A tank filled with hot water drains by gravity into a continually stirred colder tank and that tank in turn drains to the diffuser. While the diffuser floats upwards, it fills the test section creating a linear stratification of the fluid, cold 


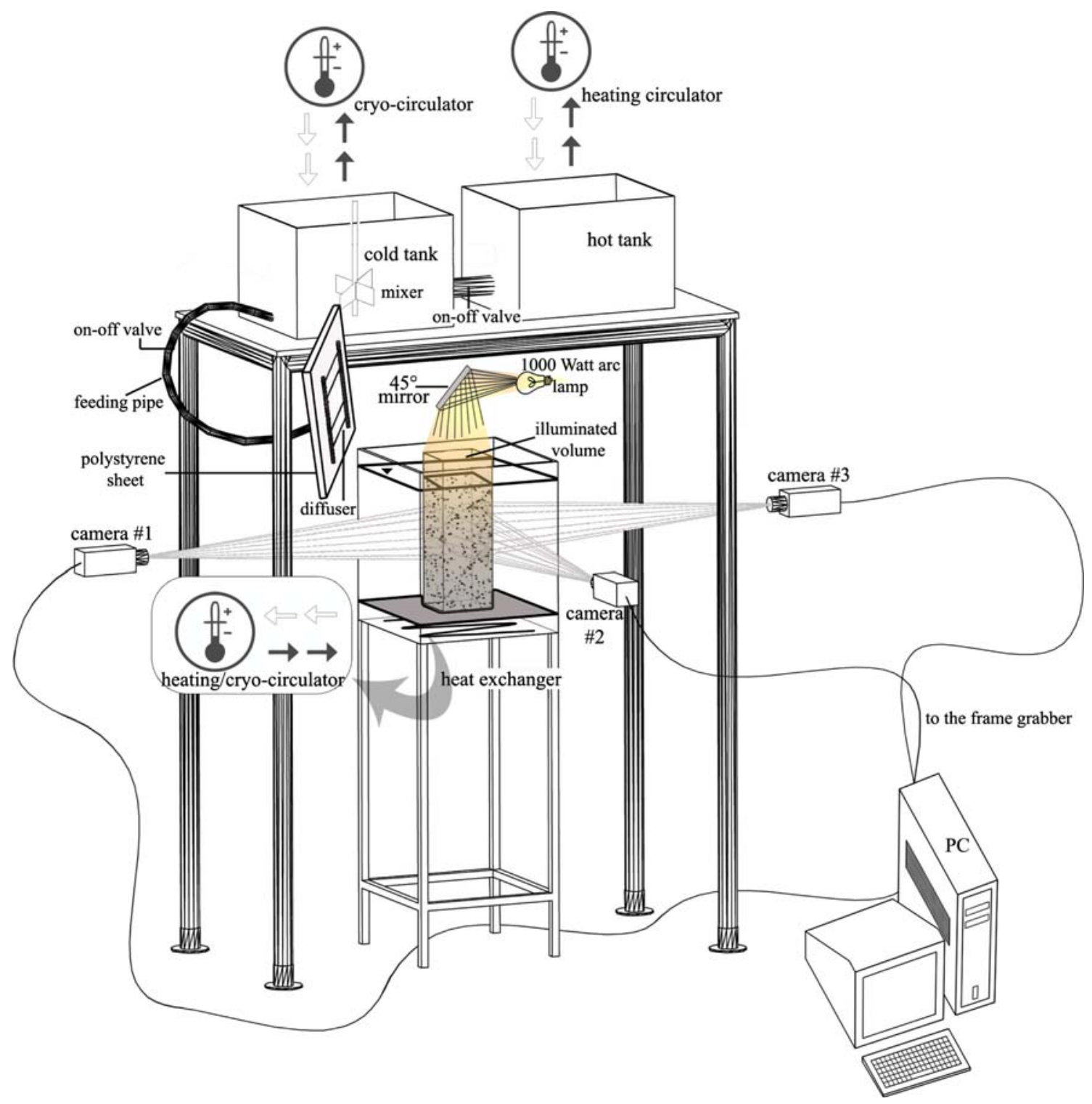

Fig. 1 Sketch of the experimental set-up

to hot from bottom to top (two-tank filling method). Twenty-six thermocouples are spaced vertically in the tank to record temperature. After the fluid stratification within the tank is completed, a cryostatically controlled hot water bath is attached to the metal base plate and the experiment begins. An approximately $0.15 \times 0.15 \times 0.40 \mathrm{~m}^{3}$ light volume produced by a high power lamp $(1,000 \mathrm{~W})$ is employed to illuminate the central region of the test section (shaded region inside the test section in Fig. 1). Images of the pollen particles are recorded using three synchronized $764 \times 576$ pixels CCD cameras with a time resolution of 25 frames/s. Synchronization of the cameras is obtained using the sync signal from one of the cameras as the triggering signal for the others. Each camera is connected to a different color input of a RGB frame grabber and the full resolution images are stored as single channel bmp files.

Two experiments are presented here (Table 1). Figure $2 \mathrm{a}$ displays the initial temperature profiles before heating starts. The measured profiles overlay to a linear interpolation that is very close to the experimental data in both cases. When heating from below begins, the temperature profile changes with time as the phenomenon evolves (Fig. 3). Vertical temperature profiles reveal the growth of the mixing layer height with time $\left(z_{i}(t)\right)$ (Fig. 2b).

Three regions characterize each profile. The region at the bottom boundary presents a negative gradient related to the existence of the unstable thermal boundary layer. Then the profile has a relatively uniform temperature, $\bar{T}(t)$, 
Table 1 Details of initial condition for experiments \#1 and \#2

\begin{tabular}{llll}
\hline Experiment \# & $T_{\mathrm{b} 0}(\mathrm{~K})$ & $T_{\mathrm{bC}}(\mathrm{K})$ & $\begin{array}{l}\gamma=(\partial \bar{T} / \partial z) \\
(\mathrm{K} / \mathrm{m})\end{array}$ \\
\hline 1 & 286.65 & 308.15 & 101.16 \\
2 & 293.12 & 308.15 & 55.35 \\
\hline
\end{tabular}

$T_{\mathrm{b} 0}$ is the temperature of the bottom before heating starts, $T_{\mathrm{bC}}$ is the final heating temperature and $\gamma$ is the temperature gradient

the mixing layer. Finally, above the mixing region, the temperature profile maintains the initial stratification. The temperature profile in the stable layer is not noticeably affected by the growing mixing layer. Each temperature profile is associated with a time given in the legend even though it was obtained through averaging temperature data acquired for $20 \mathrm{~s}$ at each thermocouple location. $\bar{T}(t)$ is related to $z_{i}(t)$ through the following relation:

$z_{i}(t)=\frac{1}{\gamma}\left(\bar{T}(t)-T_{\mathrm{b} 0}\right)$

Knowing the mean temperature within the mixing layer, the height can, therefore, be calculated (Fig. 2b). As we can clearly see from Fig. 2, the vertical temperature gradient plays a fundamental role in the mixing layer dynamics. Even if the heating temperature $T_{\mathrm{bC}}$ is the same for both experiments, a greater temperature gradient (exp \#1) provides greater resistance to the convective region, resulting in a lower mixing layer height at each time step.
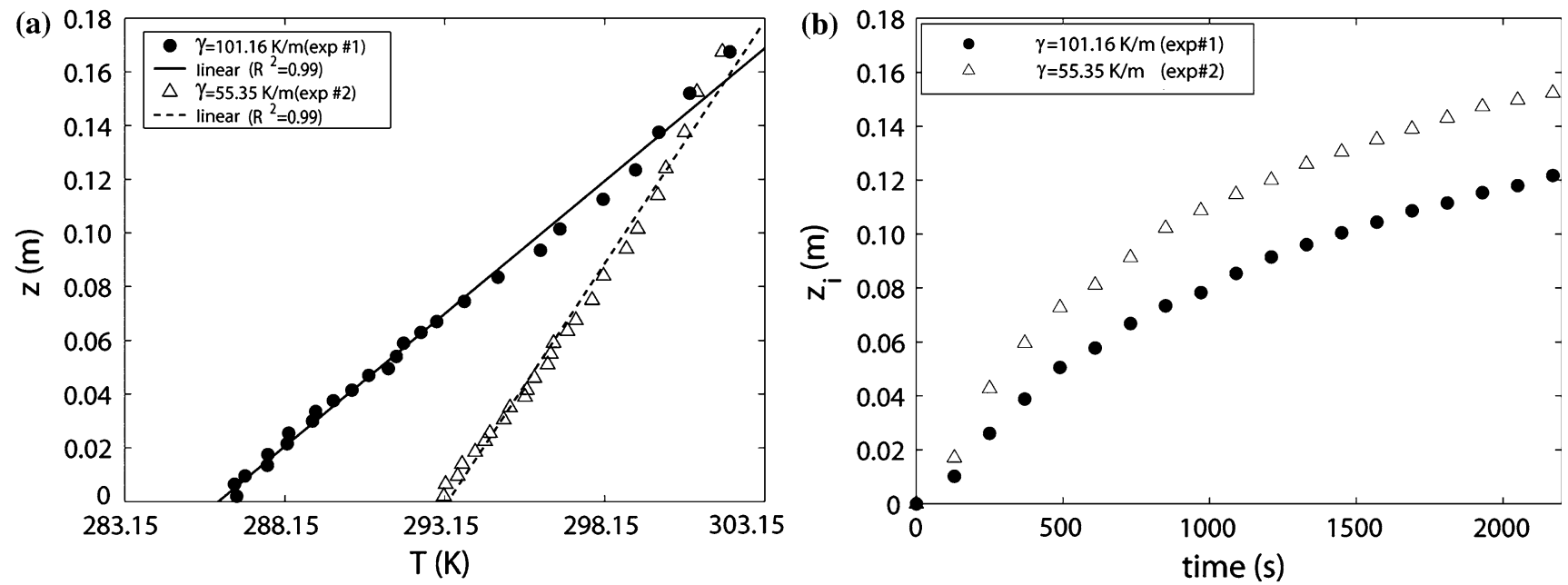

Fig. 2 a Initial temperature profiles and $\mathbf{b}$ temporal evolution of the mixing layer height $\left(z_{i}(t)\right)$ for experiments \#1 and \#2
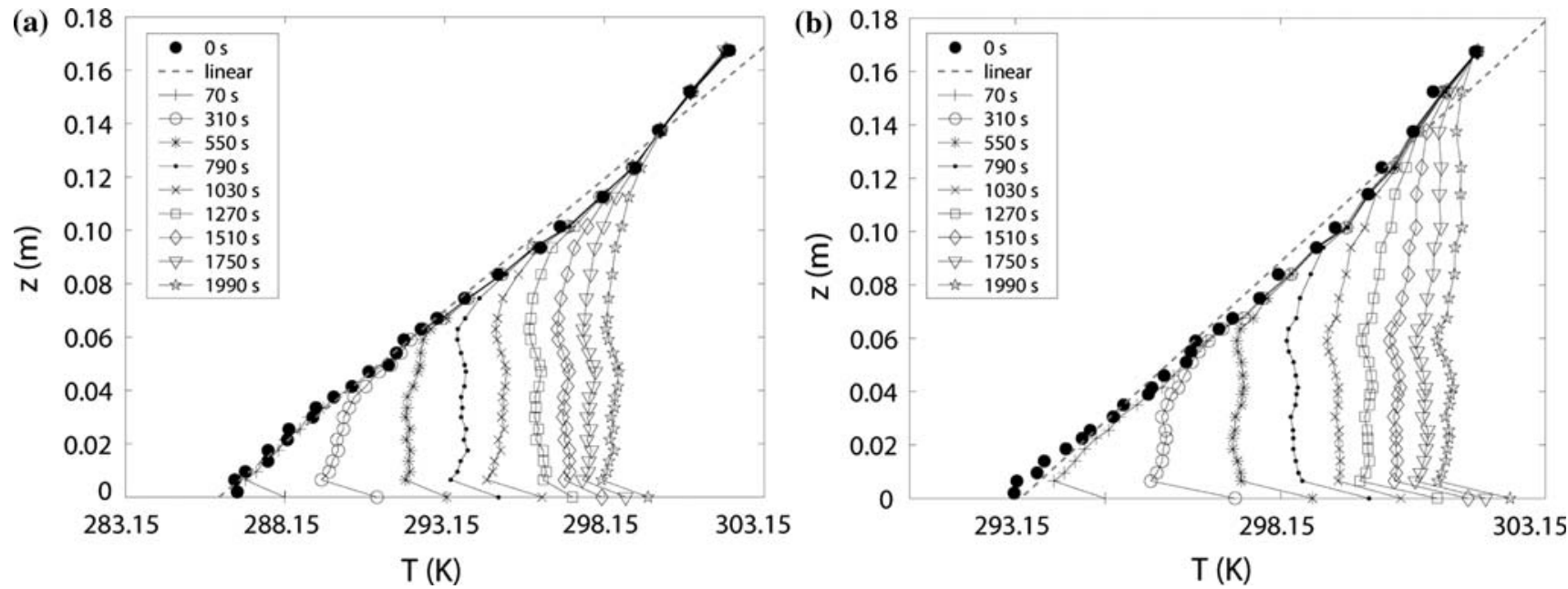

Fig. 3 a Vertical temperature profiles for experiment \#1 and $\mathbf{b}$ experiment \#2 


\section{Photogrammetric particle tracking}

The geometrical features of the experimental apparatus determine the camera arrangement; three CCD cameras were mounted as equidistant as possible from the test section with each optical axis approximately perpendicular to the side of the tank the camera faces. Subsequently, with a square-based tank, the optical axes of successive cameras should be at $90^{\circ}$ angles and lie on the same plane.

The starting points for the photogrammetric reconstruction of a 3D object in real space from 2D image projections are: the pinhole model for cameras and the mathematical relations of photogrammetry based on the collinearity assumption. According to the pinhole principle, a camera is modeled by its projective center $C$ and the image plane $\pi$. A 3D point $P$ is projected into an image point $P^{\prime}$ given by the intersection of $\pi$ with the line containing $C$ and $P$. The line containing $C$ and orthogonal to $\pi$ is called the optical axis and its intersection with $\pi$ is the principal point $(P P)$. The distance between $C$ and $\pi$ is the focal distance $(c)$.

Introduce the following reference frames (Fig. 4):

- The world reference frame $O X Y Z$ is an arbitrary 3D reference frame where the position of $3 \mathrm{D}$ points in the scene is expressed and can be directly measured.

- The image reference frame $\xi \eta \zeta$ is the coordinate system where the position of pixels in the image is expressed ( $\zeta=0$ for all image point and $\zeta=c$ for the projective center).
- The camera standard reference frame Cxyz is a 3D reference frame solidal to the camera, centered in $C$, with the $z$ axis coincident with the optical axis, $x$ parallel to $\xi$ and $y$ parallel to $\eta$.

According to these reference frames, the fundamental relations of photogrammetry allow one to determine the image coordinates $\xi$ and $\eta$ of a generic image point $P^{\prime}$ from the $X, Y, Z$ coordinates of the corresponding object point $P$ in the world reference frame, knowing the camera space orientation and its intrinsic parameters. Starting from the collinearity condition, which states that the object point, camera projective center and image point must lie on a straight line (Maas 1992), the relations are analytically described by Eqs. 2 and 3:

$$
\begin{aligned}
& \xi=\xi_{0}-c \frac{r_{11}\left(X-X_{0}\right)+r_{21}\left(Y-Y_{0}\right)+r_{31}\left(Z-Z_{0}\right)}{r_{13}\left(X-X_{0}\right)+r_{23}\left(Y-Y_{0}\right)+r_{33}\left(Z-Z_{0}\right)} \\
& \eta=\eta_{0}-c \frac{r_{12}\left(X-X_{0}\right)+r_{22}\left(Y-Y_{0}\right)+r_{32}\left(Z-Z_{0}\right)}{r_{13}\left(X-X_{0}\right)+r_{23}\left(Y-Y_{0}\right)+r_{33}\left(Z-Z_{0}\right)}
\end{aligned}
$$

$(X, Y, Z)$ object point coordinates in the world reference frame,

$\left(X_{0}, Y_{0}, Z_{0}\right)$ camera projective center coordinates in the world reference frame,

$r_{i j} \quad$ elements of $3 \times 3$ rotation matrix $\mathbf{R}$ with angles $\omega, \phi, \kappa$,

$\left(\xi_{0}, \eta_{0}\right) \quad$ image principal point,

c image focal distance.
Fig. 4 Sketch of the calibration procedure with detail of reference frames (from Maas et al. 1993)

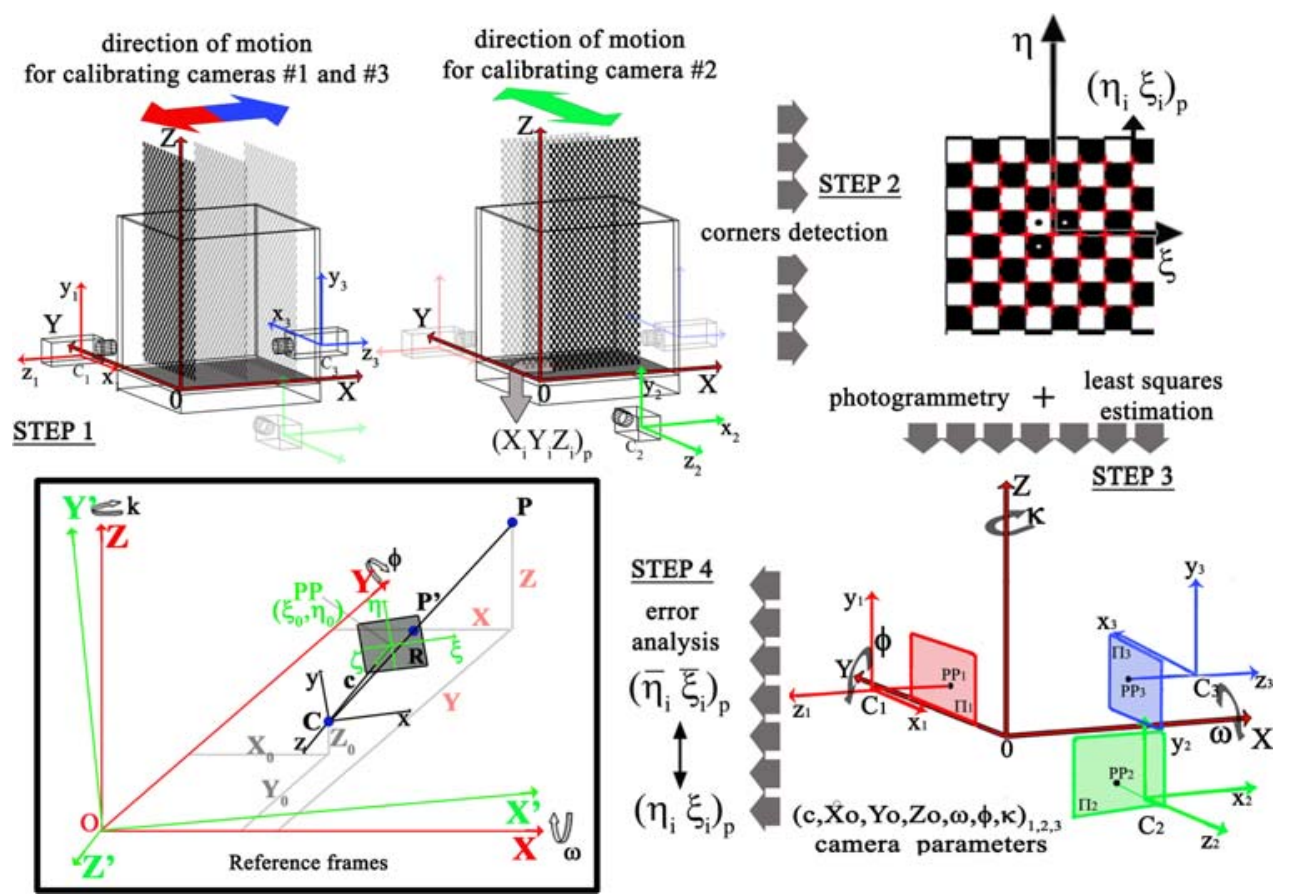


The spatial rotation matrix $\mathbf{R}$ indicates the spatial arrangement of the frame related to the coordinate system $O X Y Z$. The elements $r_{i k}$ can be expressed as a function of the rotations $\omega, \phi$ and $\kappa(4)$ :
Pre-calibration was performed to ensure each camera's optical axis is approximately orthogonal to the correspond-

$\mathbf{R}_{\omega \phi \kappa}=\left(\begin{array}{lll}\cos \phi \cos \kappa & -\cos \phi \sin \kappa & \sin \phi \\ \cos \omega \sin \kappa+\sin \omega \sin \phi \cos \kappa & \cos \omega \cos \kappa-\sin \omega \sin \phi \sin \kappa & -\sin \omega \cos \phi \\ \sin \omega \sin \kappa-\cos \omega \sin \phi \cos \kappa & \sin \omega \cos \kappa+\cos \omega \sin \phi \sin \kappa & \cos \omega \cos \phi\end{array}\right)$

where $\omega$ is the rotation in a counter clockwise direction around the $X$ axis, $\phi$ around the $Y$ axis and $\kappa$ around the $Z$ axis in order to have $X, Y$ and $Z$ overlapping $x, y$ and $z$, respectively. The order the rotations have to be applied is $\omega, \phi$ and $\kappa$.

Parameters to be determined in order to use Eqs. 2 and 3 are the camera intrinsic parameters $\left(\xi_{0}, \eta_{0}, c\right)$ and extrinsic parameters $\left(X_{0}, Y_{0}, Z_{0}, \omega, \phi, \kappa\right)$. The camera manufacturer states that $\xi_{0}$ and $\eta_{0}$ are zero. System calibration is needed to deduce the unknown parameters. Additional parameters for systematic image deformation and the multimedia geometry are not included in the calibration procedure, but calibration was conducted both inside water and air to directly check the effects of a multimedia geometry and include those effects into the calibration parameters.

We have chosen a world coordinate reference system $(O X Y Z)$ with its origin coincident with the test section lower internal corner. Considering the point of view of camera 2, the reference system axes are parallel to the three edges of the test section with the $X$ axis oriented from left to right, the $Z$ axis from the bottom to the top and the $Y$ axis inside the tank (Fig. 4).

\subsection{Calibration}

The aim of the calibration procedure for a stereoscopic system is to determine the intrinsic and extrinsic parameters of each camera in order to solve the photogrammetric problem. In many cases, the overall performance of the structure-fromstereo reconstruction step strongly depends on the accuracy of the camera calibration, a very sensitive procedure. Several methods for geometric camera calibration are presented in the literature, divided into explicit, based on the above physical parameters, and implicit methods, where the physical parameters are replaced by a set of non-physical implicit parameters that are used to interpolate between some known target points (Heikkila and Silven 1997).

We developed an explicit physically based calibration procedure using Eqs. 2 and 3 in their inverse form and minimizing a non-linear error function through least squares estimation. ing test section face, providing the starting values of the rotation angles for the calibration procedure (see Table 2).

The following steps allow calibration of the acquisition system (Fig. 4):

1. place a equally spaced $0.01 \mathrm{~m}$ grid of alternatively distributed black and white squares inside the test section;

2. mount this target on a precision motor positioner, which can move along two perpendicular directions;

3. for each camera acquire images of the target for seven different positions, each parallel to the others and to the corresponding face of the test section, along the optical axis direction of the considered camera; the coordinates of each target point in the world reference frame $\left(X_{i} Y_{i} Z_{i}\right)$ are measured with an uncertainty of $0.1 \mathrm{~mm}$ (step 1 in Fig. 4);

4. reconstruct the target point coordinates in the image reference frame $\left(\xi_{i} \eta_{i}\right)$ using a corner detection algorithm (step 2 in Fig. 4);

5. convert the image coordinates from pixels to centimeters using the camera sensible area dimensions (for our $\mathrm{CCD} 0.64906 \times 0.48306 \mathrm{~cm}^{2}$ corresponds to $782 \times$ 582 pixels);

6. identify the camera intrinsic and extrinsic orientation parameters in the absolute reference system using the photogrammetric approach (step 3 in Fig. 4; Moroni et al. 2009);

7. compute the estimated coordinates $\left(\bar{\xi}_{i}, \bar{\eta}_{i}\right)$ using the photogrammetric equations, together with the calibration parameters found in the previous step, and perform the error analysis (step 4 in Fig. 4).

Table 2 Data related to the calibration procedure

\begin{tabular}{|c|c|c|c|c|c|c|c|c|}
\hline & \multicolumn{4}{|c|}{ Water calibration } & \multicolumn{4}{|c|}{ Air calibration } \\
\hline & $N$ points & $\omega_{\mathrm{st}}$ & $\phi_{\mathrm{st}}$ & $\kappa_{\mathrm{st}}$ & $N$ points & $\omega_{\text {st }}$ & $\phi_{\mathrm{st}}$ & $\kappa_{\mathrm{st}}$ \\
\hline Camera \#1 & 2,225 & 90 & 270 & 0 & 2,375 & 90 & 270 & 0 \\
\hline Camera \#2 & 2,246 & 90 & 0 & 0 & 2,451 & 90 & 0 & 0 \\
\hline Camera \#3 & 2,230 & 90 & 90 & 0 & 2,429 & 90 & 90 & 0 \\
\hline
\end{tabular}


The three-camera set-up of Fig. 4 involves the following rotation matrices computed from the starting values of $\omega, \phi$ and $\kappa$ reported in Table 2 (for camera \#1, camera \#2 and camera \#3, respectively):

$$
\begin{aligned}
\mathbf{R}_{1}= & {\left[\begin{array}{ccc}
0 & 0 & -1 \\
-1 & 0 & 0 \\
0 & 1 & 0
\end{array}\right], \quad \mathbf{R}_{2}=\left[\begin{array}{ccc}
1 & 0 & 0 \\
0 & 0 & -1 \\
0 & 1 & 0
\end{array}\right], } \\
\mathbf{R}_{3} & =\left[\begin{array}{lll}
0 & 0 & 1 \\
1 & 0 & 0 \\
0 & 1 & 0
\end{array}\right]
\end{aligned}
$$

We assume the pre-calibration is unreliable and the rotation matrix angles are unknowns. For each camera, Table 2 displays the number of points employed for the calibration procedure.

The final calibration parameters are obtained by applying an iterative procedure based on least squares. To make sure the minimum residual reached is not local, various initial values are used to ensure the results that do not vary.

Figure $5 \mathrm{a}$ and $\mathrm{b}$ provides a qualitative representation of the quality of the calibration procedure by presenting the scatter plots of the difference between the expected and measured image coordinates, $\bar{\xi}-\xi$ and $\bar{\eta}-\eta$, versus the expected image coordinates, $\bar{\xi}$ and $\bar{\eta}$, for one of the seven views acquired by the camera \#2. To quantitatively evaluate the reliability of the entire calibration procedure, the statistics (mean and standard deviation) of the distance between expected and measured positions of grid points have been computed and results are reported in Table 3 . Note that the mean distance is nearly always less than one pixel.

Table 3 shows the results of calibration conducted both in water and in air; one can clearly see that water acts as a lens artificially increasing the effective focal lengths (for

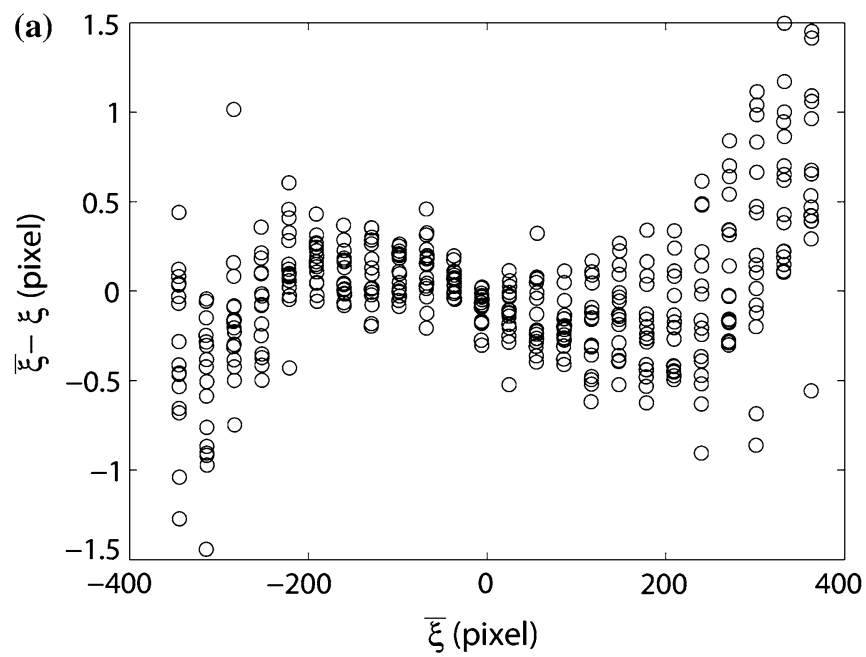

Fig. 5 a Difference between the expected image coordinate $\bar{\xi}$ and the measured image coordinate $\xi$ versus $\bar{\xi}$ for one of the seven views acquired by camera $\# 2$; b difference between the expected image camera \#1 the focal distance is $0.0354 \mathrm{~m}$ in water and $0.0264 \mathrm{~m}$ in air) and the distance between each camera's projective center and the tank face orthogonal to the correspondent optical axis (the coordinates $Y_{0}$ and $Z_{0}$ of camera \#1 remain substantially unchanged while $X_{0}$ is $-1.0606 \mathrm{~m}$ in water and $-0.7889 \mathrm{~m}$ in air, i.e. when air fills the test section instead of water, the camera apparently translates along its optical axis reducing its distance from the test section).

\subsection{Structure-from-stereo}

The purpose of the structure-from-stereo step is the reconstruction of the 3D geometry in a scene from two or more views taken with pinhole cameras. Two processes are involved: correspondence or matching and reconstruction. Correspondence implies estimating which points in the images are projections of the same scene point. Two cameras are enough to reconstruct a 3D image, but additional views increase confidence in the results. The coordinates of corresponding points are related by the epipolar geometry. Assuming the orientation parameters of the cameras are known from the calibration procedure, given a point in one image, its conjugate must belong to a line in the other image, the epipolar line (Fig. 6).

Reconstruction recovers the full 3D coordinates of points using the estimated correspondences and intrinsic and extrinsic calibration parameters.

The two most important processing steps in 3D-PTV (i.e. the establishment of spatial and temporal correspondences between particle images in simultaneous acquisitions of multiple cameras) can be handled in different ways:

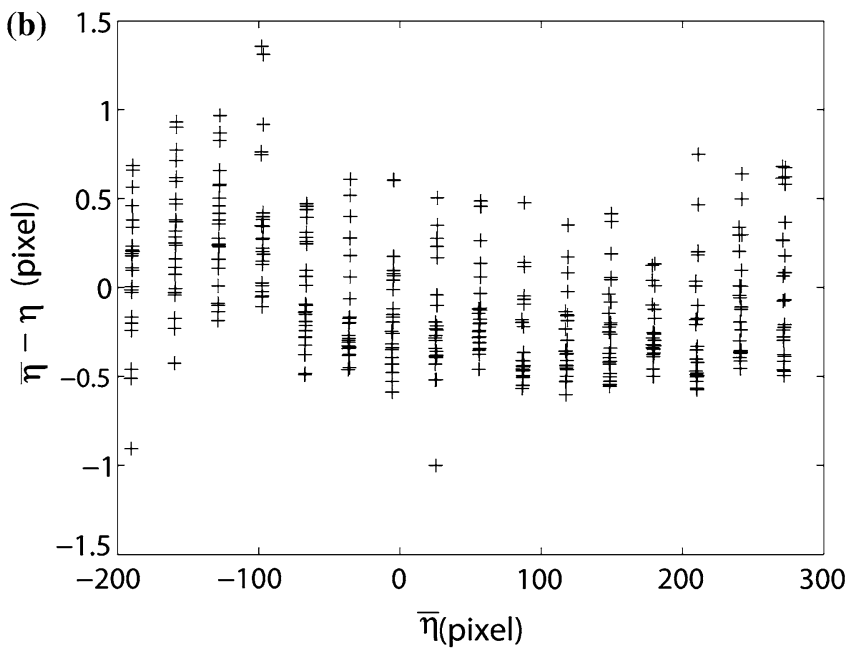

coordinate $\bar{\eta}$ and the measured image coordinate $\eta$ versus $\bar{\eta}$ for one of the seven views acquired by camera \#2 
Table 3 Results of the calibration procedure for calibration inside water and air (we use a $25 \mathrm{~mm}$ lens for each camera)

\begin{tabular}{|c|c|c|c|c|c|c|c|c|c|}
\hline Camera & $X_{0}(\mathrm{~m})$ & $Y_{0}(\mathrm{~m})$ & $Z_{0}(\mathrm{~m})$ & $c(\mathrm{~m})$ & $\omega$ & $\phi$ & $\kappa$ & $\begin{array}{l}\text { Mean distance } \\
\text { (pixel) }\end{array}$ & $\begin{array}{l}\text { SD } \\
\text { (pixel) }\end{array}$ \\
\hline \multicolumn{10}{|c|}{ Water calibration } \\
\hline$\# 1$ & -1.0606 & 0.1873 & 0.0775 & 0.0354 & 89.99 & 269.68 & -0.06 & 0.72 & 0.35 \\
\hline$\# 2$ & 0.1967 & -1.0630 & 0.0721 & 0.0360 & 90.23 & -0.14 & 0.08 & 0.64 & 0.38 \\
\hline$\# 3$ & 1.5065 & 0.1748 & 0.0659 & 0.0361 & 90.10 & 89.76 & -0.008 & 1.57 & 0.29 \\
\hline \multicolumn{10}{|c|}{ Air calibration } \\
\hline$\# 1$ & -0.7889 & 0.1855 & 0.0777 & 0.0264 & 89.96 & 269.59 & -0.08 & 0.47 & 0.29 \\
\hline$\# 2$ & 0.1969 & -0.7873 & 0.0744 & 0.0266 & 90.16 & -0.11 & 0.06 & 0.51 & 0.31 \\
\hline$\# 3$ & 1.2458 & 0.1754 & 0.0655 & 0.0273 & 90.13 & 89.69 & -0.01 & 2.24 & 0.76 \\
\hline
\end{tabular}

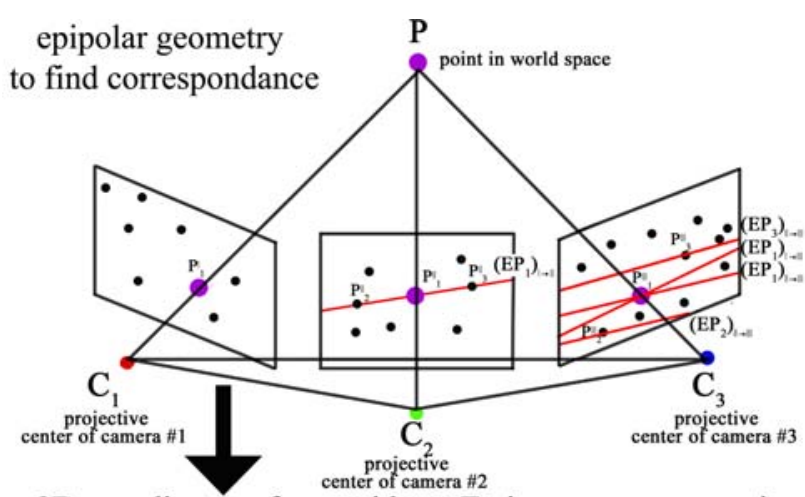

3D coordinates of centroids Trajectory reconstruction

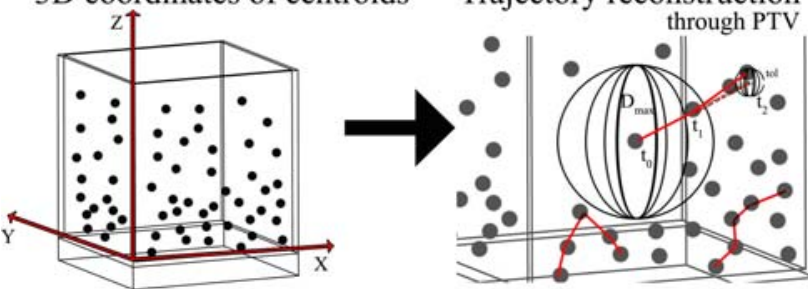

Fig. 6 Sketch of correspondence and reconstruction procedure, used to lengthen 3D trajectories $\left(P_{i}^{j}\right.$ refers to the $i$ th candidate of point $P$ on the $j$ th view; $\left(E P_{i}\right)_{j-k}$ is the epipolar line of $P_{i}$ on view $k$ from view $j$, where $i=1,2,3$ and $j=k=\mathrm{I}$, II, III; $D_{\max }$ is the radius of the sphere where the algorithm looks for all centroids at time $t_{1}$ dictated by the maximum expected value of the velocity field, while tol is the radius of the sphere where the algorithm looks for all centroids at time $t_{2}$ dictated by the local acceleration)

- 3D particle positions determined for each time step and tracking performed in 3D space (Malik et al. 1993; Kasagi and Nishino 1990);

- particles tracked in image space and establishment of spatial correspondences between 2D trajectories (Moroni and Cushman 2001);

- combination of image- and object space-based information to establish spatio-temporal correspondences between particle positions at consecutive time steps (Willneff and Gruen 2002).

The first method is employed in the present work. The main advantage in tracking 3D positions as opposed to tracking the projections in image space is positions may appear to coincide or cross, and tracking ambiguities increase in the projected image. The algorithm that reconstructs 3D trajectories employs the classical geometric method and if ambiguities arise, the nearest neighbor principle ("minimum acceleration" criterion) is employed (Moroni and Cenedese 2005).

\section{Results}

\subsection{Application to synthetic images}

The matching algorithm was tested on a synthetically generated data set simulating curling trajectories in a volume of dimensions similar to the ones employed for the experiments (Fig. 7).

The starting location of each trajectory is randomly distributed in a region close to the bottom of the observation volume, particles are assumed to remain in the lighted volume and the mean velocity is constant for each trajectory. Particles are allowed to move along the positive direction of the $Z$ axis. The synthetic trajectory lengths (in SI units) are similar to those expected in the experiments.

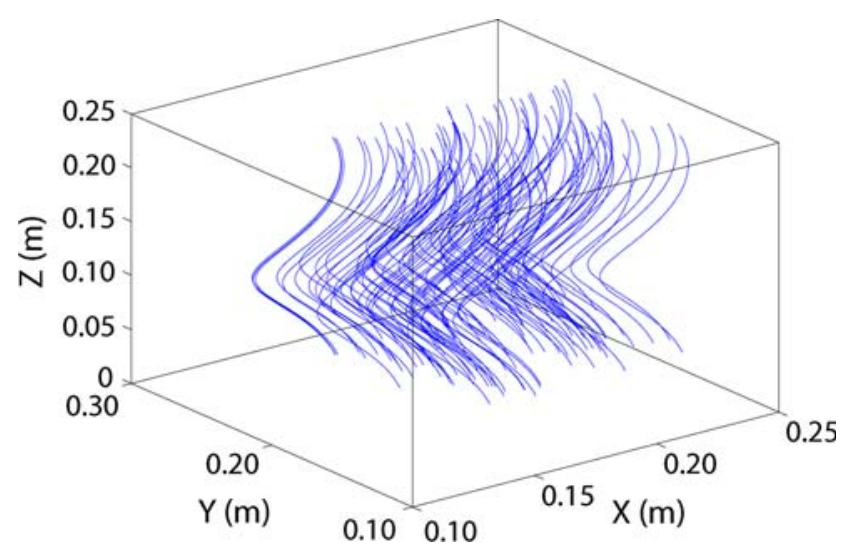

Fig. 7 Synthetically generated data set simulating curling trajectories 
The effect of increasing the number of particles seeding the measurement volume (from 100 particles to 2,000 particles) on the matching procedure was tested.

3D-PTV requires viewing the sample volume from two or more directions. The performance of the matching algorithms has been tested using both 2 and 3 cameras. The ideal set-up for obtaining highly accurate trajectories (Becker et al. 1999) requires the cameras to be mounted with the distance between them equal to the distance to the center of the measurement volume. However, the camera arrangement is usually a compromise between ideal geometrical conditions in the measuring volume and practical restrictions associated with the experiment. For this reason, three different three-camera set-ups (labeled A, B and C) have been tested (Fig. 8). Arrangement A corresponds to a three-camera set-up with optical axes lying on the same plane and forming an angle of $90^{\circ}$ with the closer camera (the arrangement is employed in the present set of measurements); Arrangement B presents three cameras with optical axes lying on the same plane and forming an angle of $60^{\circ}$ (this arrangement is used by Moroni et al. 2009); Arrangement $\mathrm{C}$ presents three cameras with optical axes of each couple of cameras forming an angle of $90^{\circ}$ (this arrangement is used by Di Giuseppe et al. 2009).

For each arrangement, the synthetic three-dimensional trajectories have been projected on the image plane of the two (\#1 and \#2) or three cameras (\#1, \#2 and \#3) replicating the exterior and interior calibration parameters of the acquisition system employed.

The effect of a wrong evaluation of the calibration parameters was investigated as well. In this case, the projections were calculated by employing a fixed set of calibration parameters (the correct ones, "err0"), while the reconstruction was carried out using a modified set of

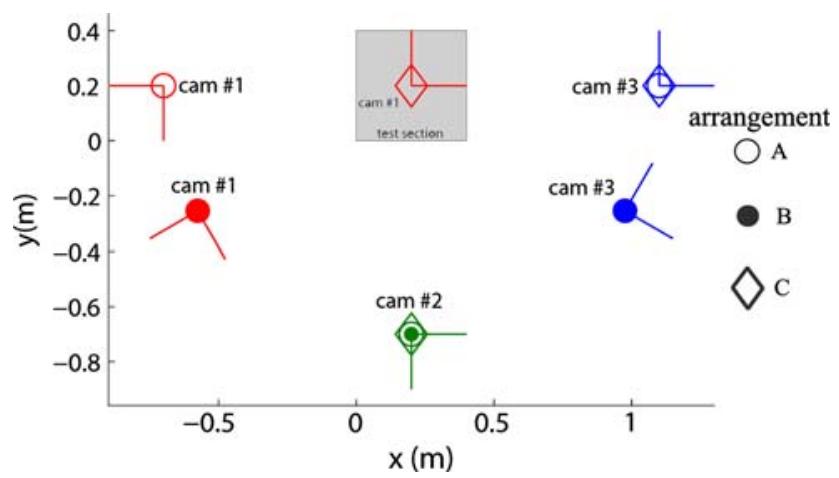

Fig. 8 Top view of the three stereo arrangements tested with synthetic trajectories. The three empty dots refer to Arrangement A, the three full dots to Arrangement $\mathrm{B}$ and the three diamonds to Arrangement $\mathrm{C}$. All camera positions are in the $(x, y)$ plane at $z=0.14 \mathrm{~m}$, except for camera \#1 of Arrangement $\mathrm{C}$ which is at $z=1.00 \mathrm{~m}$. Note that camera \#2 of the three arrangements coincides as well as camera \#3 of Arrangements A and C calibration parameters by introducing an arbitrary error in percentage ("err1" indicates a $0.01 \%$ error added, "err2" a $0.03 \%$, "err3" a $0.05 \%$, "err4" a $0.07 \%$, "err5" a $0.09 \%$ ).

Despite the small error, the projection error in pixels it determines is significant and highly dependent on the camera arrangement.

To determine the projection error, we have employed a $3 \mathrm{D}$ grid of dimensions equal to the investigated volume and known tie-point coordinates in the world reference frame. Table 4 displays the largest value of the quantity $\sqrt{\left(\Delta \xi_{i}\right)^{2}+\left(\Delta \eta_{i}\right)^{2}}$, i.e. the squared difference between "correct" and "corrupted" grid projection coordinates $(i$ refers to a generic grid point) showing the influence of the camera arrangement on the projection error. In particular, Arrangement $\mathrm{C}$ is less sensitive to the error in the calibration parameters always providing errors in pixels lower than Arrangements A and B. The error was computed with a sub-pixel accuracy but it has been reported as an integer in Table 4 (consistent with the tolerance (in pixel) introduced in the matching procedure).

The 3D positions of matched centroids output by the 3D-PTV algorithm, fed with the projections of the 3D trajectories for each arrangement, are compared to the synthetically generated data set to determine the percentage of successfully reconstructed object point locations. The tolerance may introduce ambiguities in determining the doublets (for the two-camera arrangements) or triplets (for the three-camera arrangements) corresponding to the same point in the 3D scene.

Two strategies of matching have been tested: the onechoice strategy chooses the single doublet or triplet with the smallest sum of the distances from the corresponding epipolars and epipolar intersections which satisfy the tolerance constraint in each view, the multi-choice strategy chooses all the doublets or triplets whose distances from the corresponding epipolars and epipolar intersections satisfy the tolerance criterion for each view.

The analysis of synthetic data demonstrates the correctness of the matching algorithm since it provides $100 \%$ of correctly matched doublets or triplets for all stereo arrangements, all particle densities and no error in the calibration parameters. The algorithm performances strongly decrease when the projection error in pixels becomes larger than the tolerance, set to 3 pixels in all cases.

Table 4 Projection error in pixels as a function of the stereo arrangement and the error in the calibration parameters

\begin{tabular}{lllllc}
\hline Arrangement & "err1" & "err2" & "err3" & "err4" & "err5" \\
\hline A & 2 & 5 & 8 & 11 & 15 \\
B & 2 & 6 & 9 & 12 & 16 \\
C & 1 & 2 & 3 & 4 & 5 \\
\hline
\end{tabular}


The results of the analysis of synthetic data with twocamera arrangements and one-choice strategy are synthesized in Fig. 9a. The number of doublets of the algorithm output equals the number of particles filling the observation volume for all the arrangements and set of calibration parameters employed, but the number of correctly matched doublets decreases with both increasing particle density and error in the calibration parameter set. The smallest error in the calibration parameters ("err1") yields a strong decrease in the algorithm performance for Arrangements A and $\mathrm{B}$ [i.e. when 100 particles are inserted in the observation volume, the percentage of correctly matched doublets is $19 \%$ for Arrangement A, $41 \%$ for Arrangement B and $99 \%$ for Arrangement $C$; when 200 particles are processed, 7\% (14 particles) are properly matched with Arrangement A, 19\% (38 particles) with Arrangement B and 99\% (198 particles) with Arrangement C]. This result does not change if the tolerance is increased. Arrangement $\mathrm{C}$ yields better performance of the matching algorithm, confirming it is less sensitive to error in the calibration parameters (Table 4).

It should also be noted that Arrangement $\mathrm{C}$ is more suitable to reconstruct the 3D position of our designed synthetic data set when 2 cameras are employed because of the shape of the trajectories which present similar features when projected according to Arrangements A and B but are substantially different in the Arrangement $\mathrm{C}$ because it is the only one that presents a top view of the scene. We believe this does not challenge the validity of our test because Table 4 clearly shows Arrangement $\mathrm{C}$ was influenced the least by the calibration parameter errors on the data set employed.
The results of the analysis of synthetic data with twocamera arrangements and multi-choice strategy are summarized in Fig. 9b. The number of doublets of the algorithm output is much greater than the number of particles filling the observation volume for all the arrangements and set of calibration parameters employed.

The matching algorithm performances largely improve with the percentage of correctly matched doublets always larger than $90 \%$ for errors "err1", "err2", "err3" and all arrangements. On the other hand, the number of doublets output by the matching algorithm increases by more than one order of magnitude for low particle densities (until 200 particles) and more than two orders of magnitude for high particle densities (more than 500 particles) for Arrangements $\mathrm{A}$ and $\mathrm{B}$, increasing the computational costs and producing possible ambiguities in the tracking procedure. This is clearly shown in Fig. 10a, b and c which present the difference of doublets and particles within the observation volume divided by the number of particles filling the observation volume in percentage versus the number of particles for Arrangements A, B and C, respectively, and no errors in the calibration parameters. The performance of the matching algorithms when three-camera set-ups are employed largely improves, demonstrating the need for more than two views for the correct reconstruction of a $3 D$ scene.

The results of the analysis of synthetic data with three-camera arrangements and one-choice strategy are synthesized in Fig. 11a. The number of triplets the algorithm outputs equals the number of particles within the observation volume for all the arrangements and sets of calibration parameters with an error less than the

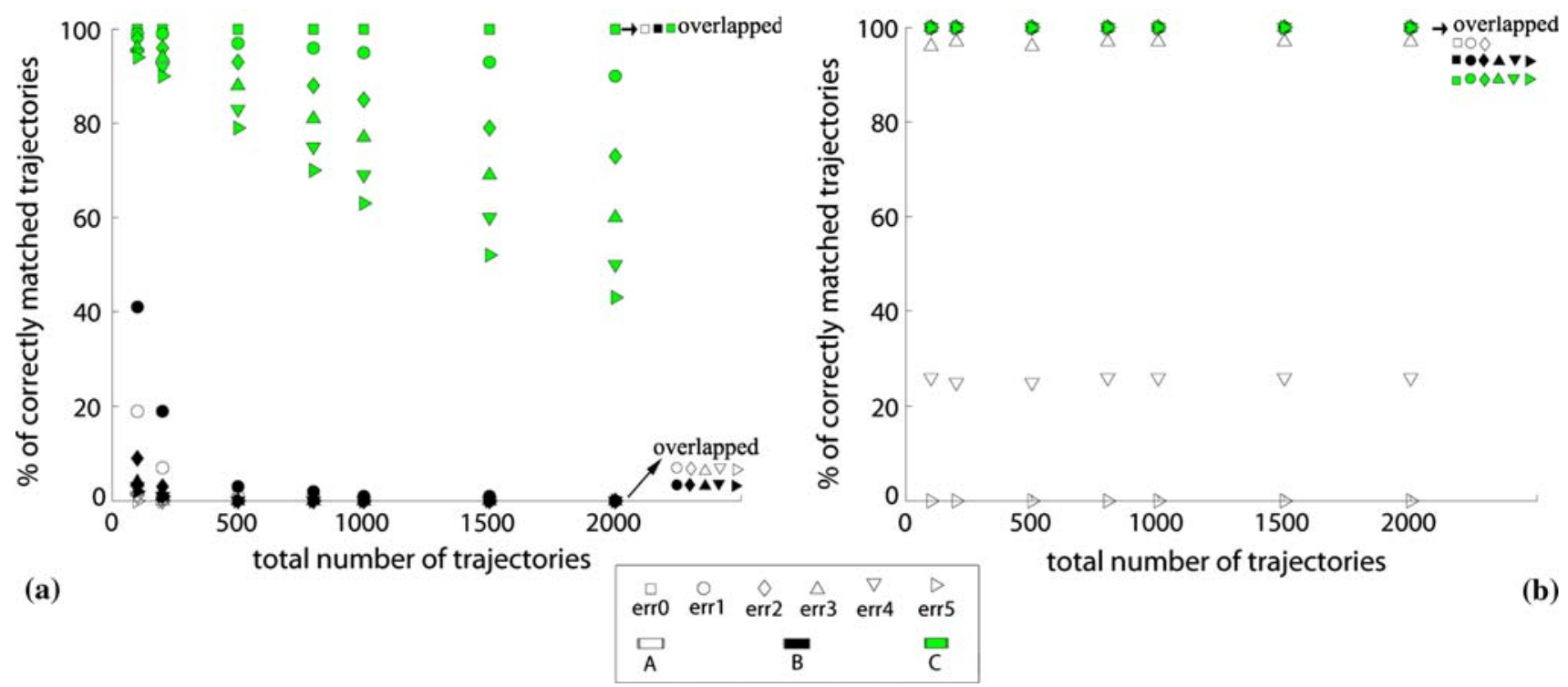

Fig. 9 Matching algorithm performances for two-camera arrangements, a one-choice strategy and $\mathbf{b}$ multiple-choice strategy 

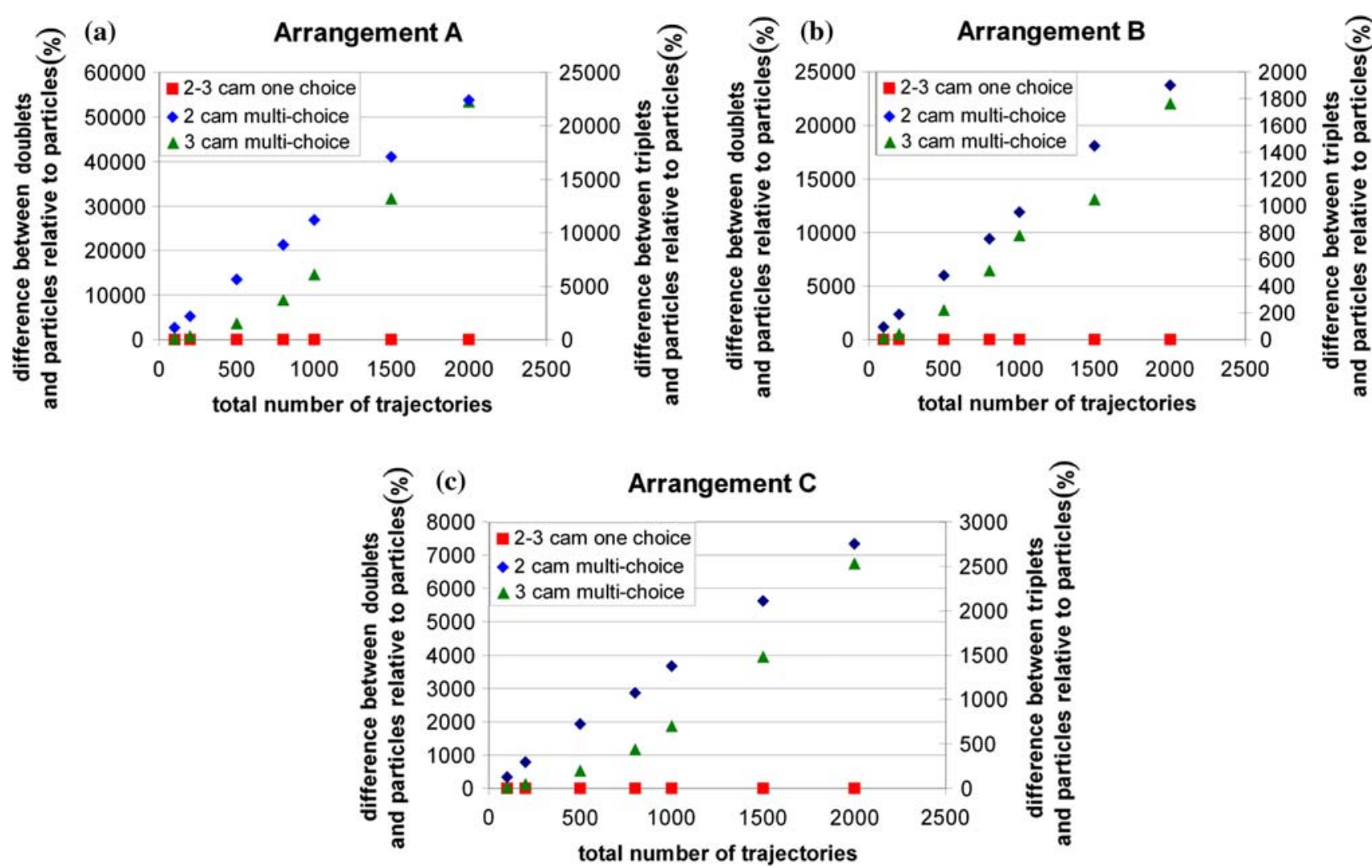

Fig. 10 Difference of doublets, or triplets, and particles within the observation volume divided by the number of particles filling the observation volume in percentage versus the number of particles for a Arrangement A, b the same for Arrangement B and $\mathbf{c}$ the same for Arrangement $\mathrm{C}$

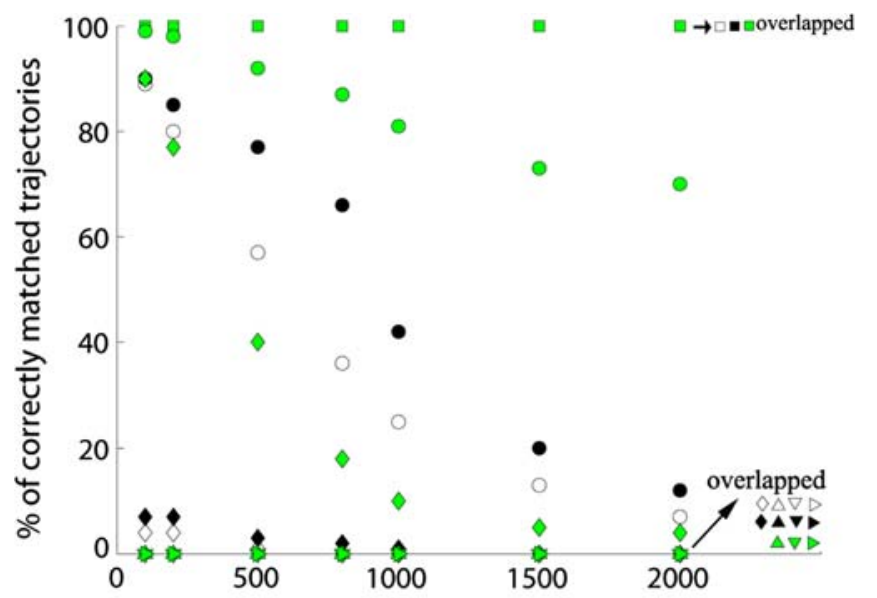

(a)

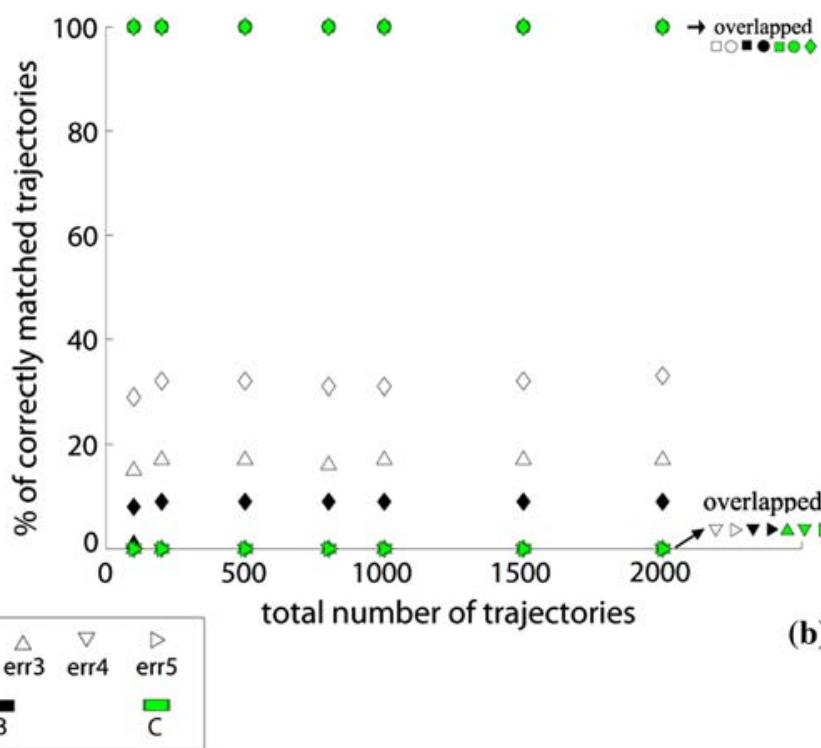

(b)

Fig. 11 Matching algorithm performances for three-camera arrangements, a one-choice strategy and b multiple-choice strategy

tolerance employed. Arrangements A and B perform better, with a percentage of correctly matched triplets always larger than the corresponding case with the twocamera arrangements. When 100 particles are inserted within the observation volume, 19 particles are correctly matched with the two-camera Arrangement A, whereas 89 are correctly matched with the three-camera Arrangement A. 
The results of the analysis of synthetic data with threecamera arrangements and multi-choice strategy are synthesized in Fig. 11b. Even in this case, the matching algorithms perform better because even if the percentage of correct triplets drops for large errors in the calibration parameter set, the significantly lower number of triplets (i.e. ambiguous particles to be tracked, see Fig. 10) output by the algorithm compensates for the lower performance if compared to the two-camera case, and suggests the introduction of a third view. On the other hand, the multi-choice strategy, compared to the one-choice strategy, always allows $100 \%$ of correctly matched triplets for small calibration errors producing projection errors in pixels lower than the tolerance. This motivates the three-camera multichoice strategy is employed to match the real data.

\subsection{Application to real images}

The stereoscopic system, properly calibrated as described in Sect. 3.1, and the matching-reconstruction algorithms, tested with synthetic data as described in the previous section, have been applied to real images to track tracer particles in penetrative convection experiments.

Figure 12 shows matched centroids projected on the $X Z$ plane (a, c, e) and the $Y Z$ plane (b, d, f) inside both the stable and the unstable layers after 220, 440 and $900 \mathrm{~s}$ from the beginning of experiment $\# 1$ and tracked for at least $50 \mathrm{~s}$. Darker colors have been linked to later times, while lighter colors to earlier times. From both projections, it can be clearly seen that the turbulent region below is moving upward against the almost quiet stable layer. The 3D-PTV procedure is suitable for reconstructing the displacement field (i.e. particle trajectories) in both the mixing and the stable layer.

The velocity data obtained from the 3D trajectories have been used to obtain quantitative results for the mixing layer height evolution. Since dispersion in turbulent convective phenomena is mostly due to transport by large organized structures, the knowledge of the vertical extension of the structures dominating the flow field, associated to the mixing layer height, is a necessary requirement (Moroni and Cenedese 2006).

The method to detect the mixing layer growth consists of reconstructing the vertical velocity standard deviation, $s_{\mathrm{w}}$, profile as a function of time employing the horizontal homogeneity hypothesis (Fig. 13).

The mixing layer region is characterized by greater values of the standard deviation of the vertical velocity than the stable layer, due to the onset of convection. For earlier times, the standard deviation is small everywhere, while, as time goes on, the standard deviation will increase in magnitude and cover an increasing portion of the fluid. This is a reflection of the growing mixing layer

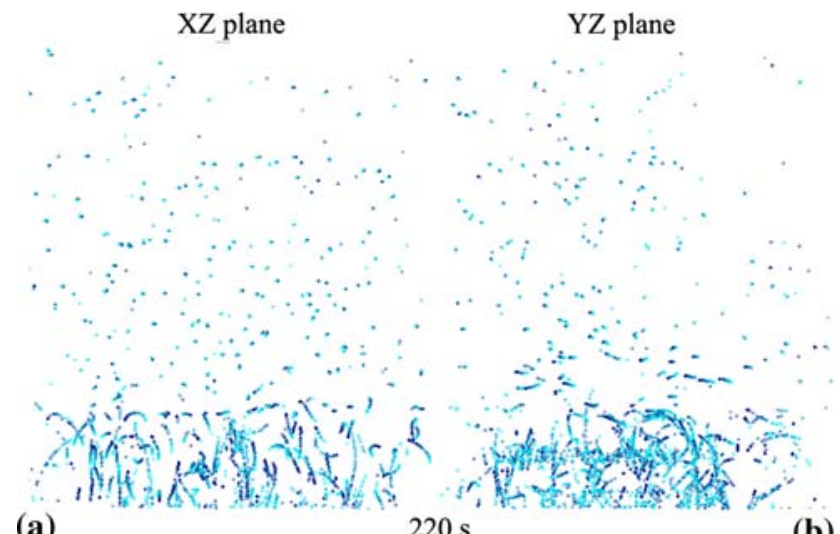

(a)

$220 \mathrm{~s}$

(b)

(c)
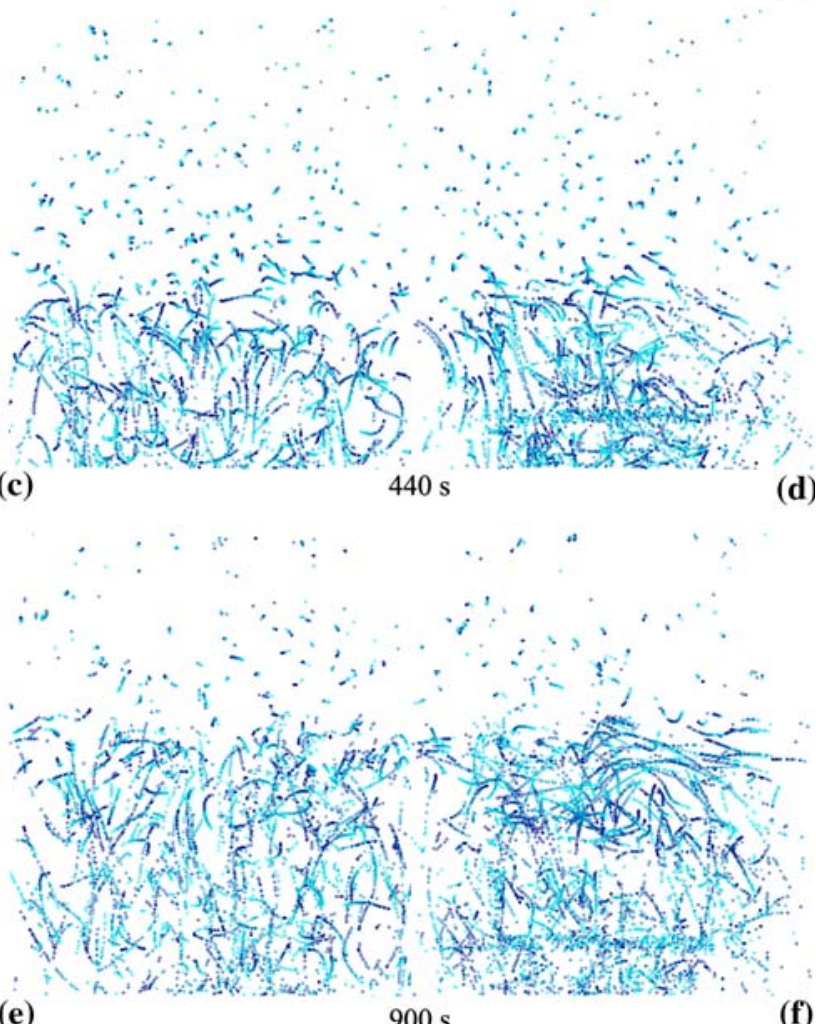

(d)

Fig. 12 Projections on the $X Z$ plane $(\mathbf{a}, \mathbf{c}, \mathbf{e})$ and $Y Z$ plane $(\mathbf{b}, \mathbf{d}, \mathbf{f})$ of matched centroids after $220 \mathrm{~s}(\mathbf{a}, \mathbf{b}), 440 \mathrm{~s}(\mathbf{c}, \mathbf{d}), 900 \mathrm{~s}(\mathbf{e}, \mathbf{f})$ from the beginning of experiment \#1. In both pictures, $50 \mathrm{~s}$ are overlaid and dark color refers to later times (exp \#1 of Table 1)

characterized by fluctuations of the velocity field about the mean value.

For each profile, we find the height where the standard deviation became, after the peak, $30 \%$ of the maximum value and the profile slopes are still gentle, thus the $z_{i}$ will be:

$z_{i}(t)=\frac{1}{k} z_{30 \%}(t)$

where $z_{30 \%}(t)$ is the height associated to the standard deviation equal to $30 \%$ of the maximum value and $k=0.7$ 


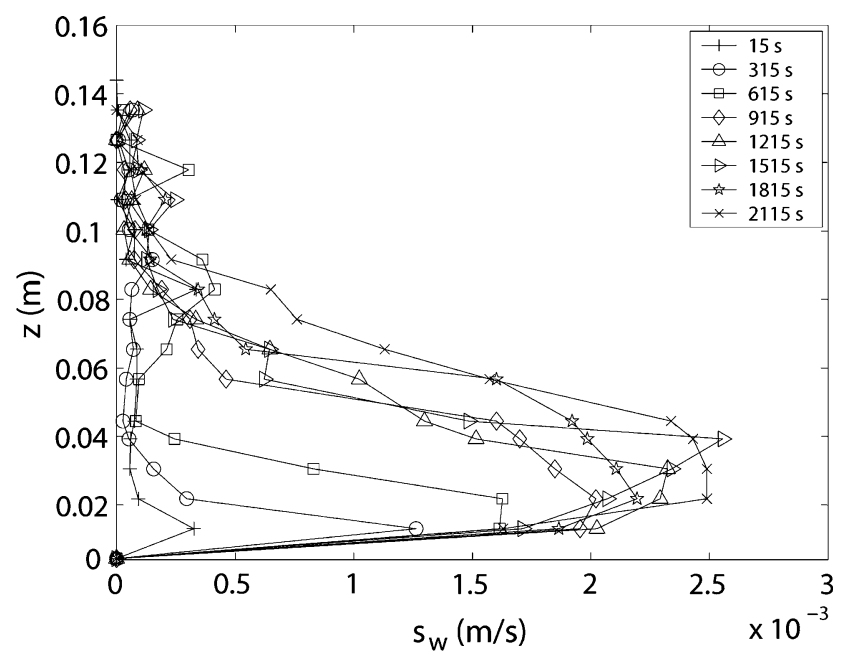

Fig. 13 Standard deviation profiles of the vertical velocity at different times for experiment \#1

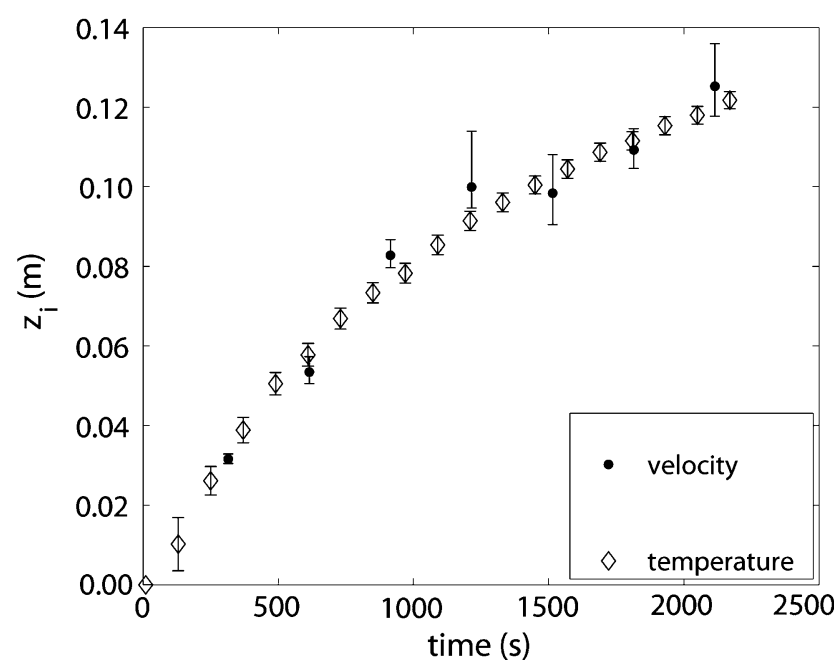

Fig. 14 Comparison of the mixing layer height evolution computed by temperature and velocity measurements

is a constant empirically determined using a larger set of experiments and findings of Cenedese and Querzoli 1994.

The mixing layer height evolution found using the method described above is plotted in Fig. 14. Results using velocity data are compared to the ones obtained using the temperature data, showing good agreement.

Error bars of $z_{i}$ are also plotted for both the two methods employed. They are computed starting from the $90 \%$ confidence interval for the vertical velocity standard deviation $\sigma_{\mathrm{w}}$ based on the sample standard deviation $s_{\mathrm{w}}$ and the Chi-square function, and from the $90 \%$ confidence interval for the mean temperature $\mu_{\mathrm{T}}$ based on the sample mean $\bar{T}$ and $t$-Student function (Bendat and Piersol 1971). The number of independent samples is computed using the autocorrelation coefficient (Dore et al. 2009).
The evaluation of $z_{i}$ using temperature data seems to be statistically more accurate for later times, but it is not for earlier times due to the low number of samples. On the contrary, the velocity-based method is very sensitive to the irregular shape of standard deviation profiles.

Figure 15 presents the standard deviation profiles normalized accordingly the Deardorff mixed layer similarity (Willis and Deardorff 1974), by employing the convective velocity, $w^{*}$, and the mixing layer height, $z_{i}$, found through temperature measurements. After normalization, all profiles collapse on the same curve, losing the dependence on time and particular conditions of the experiment. The observation of normalized profiles proves that the mixing layer upper limit is located where the standard deviation became about $7 \%$ of the maximum value. Once we prove the phenomenon is self-similar, that percentage depends neither on experiment conditions nor on time.

Our present results are also compared with atmospheric models (Lenschow 1974; Lenschow et al. 1980), tank experiments (Willis and Deardorff 1974; Deardorff and Willis 1985; Cenedese and Querzoli 1994) and field measurements (Young 1988). The comparison shows a fairly nice agreement, demonstrating the validity of our experimental investigation and its applicability for the study of the real atmospheric boundary layer.

\section{Conclusions}

Three-dimensional Particle Tracking Velocimetry allows a more realistic description of the velocity field, which occurs during the evolution of the convective mixing layer, than more traditional 2D techniques. Furthermore, photogrammetric 3D-PTV rather than "scanning" 3D-PTV (Moroni and Cushman 2001) is more accurate when the tracer particle density is high, because particles may be tracked directly in the 3D space rather than through matching of $2 \mathrm{D}$ projections.

Sensitivity tests conducted on the matching algorithm prove that the calibration accuracy is fundamental to obtain the correct matching and particle tracking; small error in calibration parameters or neglecting water refraction effects reduces matching performance. On the other hand, accuracy always less than 2 pixels is reached with our calibration procedure ensuring good results in the matching procedure.

Tests on synthetic data demonstrate that a multi-choice strategy with a three-camera arrangement is the best solution for matching data. It is less sensitive to errors in the calibration data set when both the percentage of correctly matched triplets and the number of outputs of the algorithm are considered. 

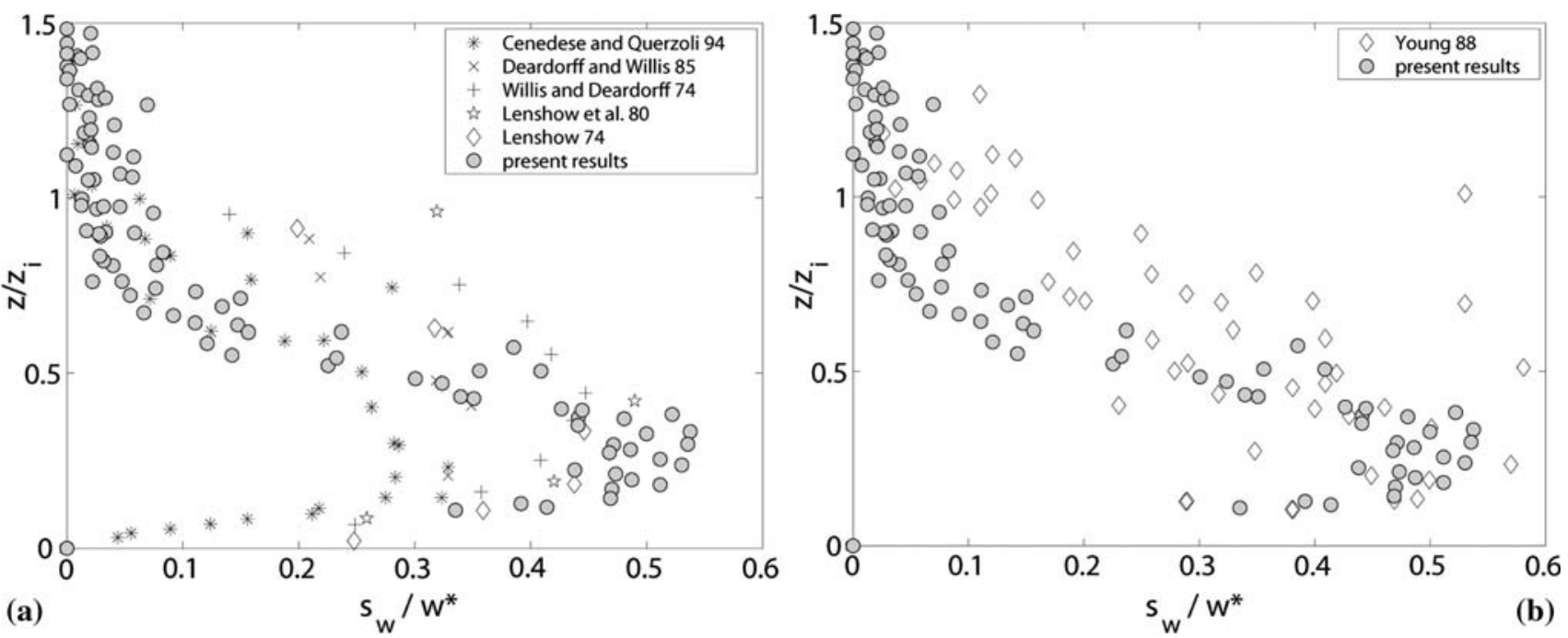

Fig. 15 Standard deviation of vertical velocity profiles normalized accordingly the Deardorff mixed layer similarity (Willis and Deardorff 1974) compared with $\mathbf{a}$ atmospheric models and tank experiments and $\mathbf{b}$ field measurements

Finally, the 3D-PTV procedure has been shown to be suitable for reconstructing the displacement field (i.e. particle trajectories) in both the mixing and the stable layer, allowing more than 1,200 particles to be tracked.

Open Access This article is distributed under the terms of the Creative Commons Attribution Noncommercial License which permits any noncommercial use, distribution, and reproduction in any medium, provided the original author(s) and source are credited.

\section{References}

Becker J, Bosemann W, Bopp R (1999) Photogrammetric methods applied to fluid motion within a fluid matrix. Meas Sci Technol 10:914-920

Bendat JS, Piersol AG (1971) Random data: analysis and measurement procedures. Wiley, New York

Cenedese A, Querzoli G (1994) A laboratory model of turbulent convection in the atmospheric boundary layer. Atmos Environ 28(11):1901-1913

Chang T, Taterson G (1983) Application of image processing to the analysis of three-dimensional flow fields. Opt Eng 23:283-287

Cowen E, Monismith S (1997) A hybrid digital particle tracking velocimetry technique. Exp Fluids 22:199-211

Deardorff JW, Willis GE (1985) Further results from a laboratory model of the convective planetary boundary layer. BoundaryLayer Meteorol 35:205-236

Di Giuseppe E, Funiciello F, Moroni M (2009) Investigating subduction mechanism through image analysis technique (in preparation)

Doh DH, Hwang TG, Saga T (2004) 3D-PTV measurements of the wake of a sphere. Meas Sci Technol 15:1059-1066

Dore V, Moroni M, Cenedese A (2009) Quantifying mixing in a convective boundary layer. Heat Trans Eng (submitted)

Guezennec YG, Brodkey RS, Trigui N, Kent JC (1994) Algorithms for fully automated three-dimensional particle tracking velocimetry. Exp Fluids 17:209-219

Haynes PH, Shuckburg EF (2000) Effective diffusivity as a diagnostic of atmospheric transport. Part II. Troposphere and lower stratosphere. J Geophys Res 105:22795-22810
Heikkila J, Silven O (1997) A four camera calibration procedure with implicit image correction. In: Proceedings of the 1997 conference on computer vision and pattern recognition (CVPR '97)

Hinsch KD, Hinrich H (1996) Three-dimensional particle velocimetry. In: Th Dracos (ed) Three-dimensional velocity and vorticity measuring and image analysis technique. Kluwer, Dordrecht

Kasagi N, Nishino K (1990) Probing turbulence with three-dimensional particle tracking velocimetry. In: Proceedings of international symposium on engineering turbulence-methods and measurements

Kieft RN, Schreel KRAM, Van der Plas GAJ, Rindt CCM (2002) The application of a 3D PTV algorithm to a mixed convection flow. Exp Fluids 33:603-611

Lenschow DH (1974) Model of the height variation of the turbulence kinetic budget in the unstable boundary layer. J Atmos Sci 31:465-474

Lenschow DH, Wyngaard JC, Pennel WT (1980) Mean-field and second moment budgets in a baroclinic, convective boundary layer. J Atmos Sci 37:1313-1326

Maas H (1992) Complexity analysis for the establishment of image correspondences of dense spatial target fields. Int Arch Photogramm Remote Sens B XXIX(5):102-107

Maas H, Gruen A, Papantoniou D (1993) Particle tracking velocimetry in three-dimensional flows. Exp Fluids 15:133-146

Malik N, Dracos T, Papantoniou D (1993) Particle tracking in threedimensional turbulent flows. Part II. Particle tracking. Exp Fluids 15:279-294

Moroni M, Cenedese A (2005) Comparison among feature tracking and more consolidated velocimetry image analysis techniques in a fully developed turbulent channel flow. Meas Sci Technol 16:2307-2322

Moroni M, Cenedese A (2006) Penetrative convection in stratified fluids: velocity measurements by image analysis techniques. Nonlinear Process Geophys 13:353-363

Moroni M, Cushman JH (2001) Three-dimensional particle tracking velocimetry studies of the transition from pore dispersion to Fickian dispersion for homogeneous porous media. Water Resour Res 37(4):873-884

Moroni M, Cushman JH, Cenedese A (2009) Application of photogrammetric 3D-PTV technique to track particles in porous media. Transp Porous Media (in press)

Stuer H, Maas HG, Virant M, Becker J (1999) A volumetric 3D measurement tool for velocity field diagnostic in microgravity experiments. Meas Sci Technol 10:904-913 
Stull RB (1988) An introduction to boundary layer meteorology. Kluwer, Dordrecht

Suzuki Y, Ikenoya M, Kasagi N (2000) Simultaneous measurement of fluid and dispersed phases in a particle-laden turbulent channel flow with the aid of 3D PTV. Exp Fluids 29:185-193

Willert CE, Gharib M (1992) Three-dimensional particle imaging with a single camera. Exp Fluids 12:353-358

Willis GE, Deardorff JW (1974) A laboratory model of the unstable planetary boundary layer. J Atmos Sci 31:297-1307
Willneff J, Gruen A (2002) A new spatio-temporal matching algorithm fro 3D-particle tracking velocimetry. In: 9th international symposium on transport phenomena and dynamics of rotating machinery. Honolulu, Hawaii, 10-14 February

Young GS (1988) Turbulence structures of the convective boundary layer. Part I. Variability of normalized turbulence statistics. J Atmos Sci 45:720-726

Zhang J, Tao B, Katz J (1997) Turbulent flow measurement in a square duct with hybrid holographic PIV. Exp Fluids 23:373-381 